\title{
Tuberculosis of the Psoas Muscle Presenting as a Mass Lesion
}

\author{
Joaquin C. Ponce-Zepeda ${ }^{\mathrm{a}}$, Moises R. Zepeda ${ }^{\mathrm{a}, \mathrm{b}}$
}

\begin{abstract}
Tuberculosis involving the psoas muscle is a rare medical phenomenon in the 21 st century. Most bacterial infections involving the psoas muscle are caused by Staphylococcus aureus followed by Streptococcus species and Escherichia coli. An abscess in the psoas muscle is clinically difficult to diagnose without the aid of modern imaging studies. However, computed tomography (CT) imaging studies and basic histopathological methods can provide an accurate and timely diagnosis. With this in mind, we report a case of a Mycobacterial infection of the psoas muscle.
\end{abstract}

Keywords: Psoas muscle; Tuberculosis; Infection; Histopathology

\section{Introduction}

A psoas abscess is considered a rare disease as reported in the medical literature. To our knowledge, the first reported cases in the United States were described by Mynter in 1881 [1]. He described two cases involving a 54- and a 28 -year-old female. Both patients had "high fevers, lumbar pain, increased pulse, and deep-seated swelling in the iliac region" [1]. Dr. Mynter clinically diagnosed an abscess, surgically drained each abscess, and reported complete recovery for each of his patients [1].

In 1992 Gruenwald cited a worldwide occurrence of 12 cases per year during the period of 1986 - 1991 [2]. While Ricci reported nine cases at his institution and his exhaustive literature review in 1986 yielded a total of 367 reported cases worldwide, since the first cases reported by Mynter [3]. These two authors found that the most common microorganisms cultured were Staphylococcus aureus, Streptococcus species, and Escherichia coli $[2,3]$.

Studies indicate that the increase in intravenous drug

Manuscript submitted November 19, 2014, accepted January 15, 2015

aDepartment of Pathology, Alhambra Hospital Medical Center, 100 S. Raymond Avenue, Alhambra, CA 91801, USA

${ }^{b}$ Corresponding Author: Moises R. Zepeda, Department of Pathology, Alhambra Hospital Medical Center, 100 S. Raymond Avenue, Alhambra, CA 91801, USA.Email: mzepeda@doctorslab.net

doi: https://doi.org/10.14740/jmc2031e abuse and HIV infection also contributes to the increased prevalence of primary iliopsoas abscess (IPA). There are two types of psoas muscle abscesses that are generally clinically identified in affected patients [4]. The first is a primary abscess that is caused by a bacteremia distant to the iliopsoas muscle, while a secondary iliopsoas abscess occurs as a result of direct spread of infection to the iliopsoas muscle by contiguity from an adjacent structure [5].

Since the days of Mynter and the clinical acumen of 19th century physicians, the clinical diagnosis of a psoas muscle abscess has been, and continues to be, extremely difficult. The clinical presentation is often vague with non-specific symptoms. Even the purported clinical triad of fever, back pain, and limp is only present in about $30 \%$ of patients $[6,7]$. This low percentage is due to the complex anatomical relationships of the iliopsoas muscle group. As most clinicians know, the iliopsoas muscle complex encompasses and originates on the edges of the 12th thoracic to the fifth lumbar vertebra spreading and inserting into the lesser trochanter of the femur, and is also directly posterior to the abdominal and retroperitoneal viscera. This specific complex anatomy contributes to the difficulty of diagnosing and treating this rare medical condition. Hence, in today's modern medicine of the 21 st century, it is best diagnosed by CT imaging studies, and it is imperative that health care providers have a high index of suspicion $[7,8]$.

In 2006, J.P. Garner conducted a 3-year study of 15 patients, all of whom presented to the hospital with pyrexia or with other inflammatory markers. Fourteen of the patients were diagnosed using a CT scan. Garner's study concluded that the incidence of IPA is most likely under-reported. His study emphasized that the vague presentation of symptoms delays diagnosis and decreases the high index of suspicion required of physicians to identity the infection early [9]. Garner also reported that magnetic resonance imaging, radionuclide imaging and positron emission tomographic imaging have all been used to evaluate IPA; however, the high cost and lack of ready availability make it difficult to argue as advantageous over CT scan [9].

Audia reviewed the radiological findings for an IPA and reported the following salient features [10]. In general, CT scans show the psoas muscle to be enlarged compared with the contralateral side with rim enhancement of the abscess wall. The CT imaging studies demonstrate a so-called "rind sign" and gas bubbles or air fluid levels are visible within the cavity. Audia also concluded that routine abdominal radiology is generally unhelpful [10].

Van den Berge et al reviewed the use of ultrasonography 


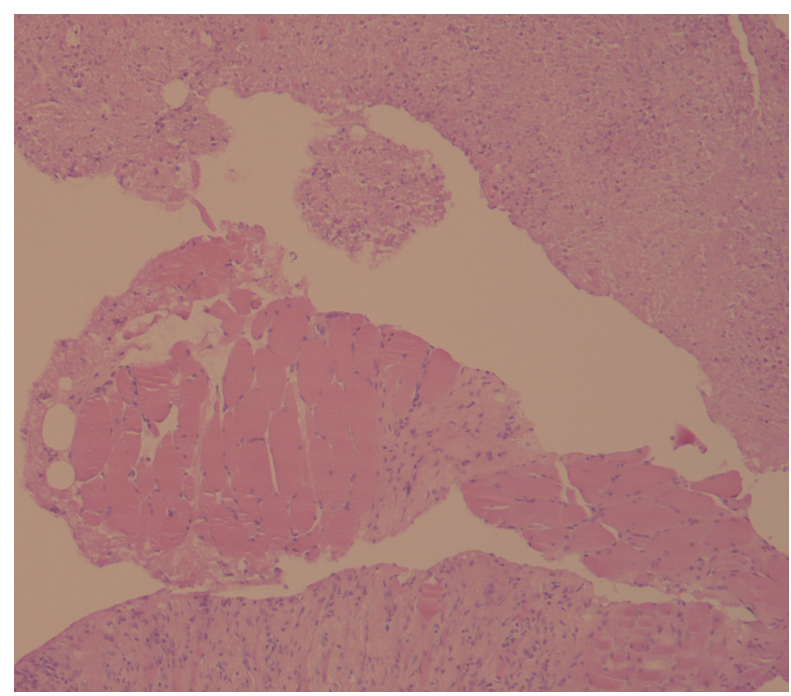

Figure 1. H\&E stain showing benign striated muscle in between striated muscle affected by granulomatous inflammation $(\times 10)$.

in diagnosing an IPA. They reported that it was useful in only $60 \%$ of cases and was highly dependent upon the operator [11]. They concluded that a CT scan is the most effective method in diagnosing an IPA.

Furthermore, a CT-guided tissue biopsy can hasten the diagnosis by histopathologic examination. Histopathology augmented with histochemistry can provide an accurate diagnosis in patients presenting with a psoas mass. Over the last century, the basic medical science of histopathology has proven to be an essential tool in the diagnosis and treatment of numerous medical and surgical conditions. The combination of CT imaging and histopathological analysis can help reduce the morbidity and mortality of this disease.

Once the psoas abscess is diagnosed, the abscess may be treated medically, surgically, or by percutaneous drainage (PCD). PCD is a minimally invasive procedure and has a success rate of up to $87 \%$ [10-12]. It is considered a safer procedure as compared to open surgical drainage. All treatment options have advantages as well as disadvantages. Choosing one over another, or a combination of different treatment modalities, should be decided on a case by case basis, and in consultation with all physicians responsible for the patient's care [10-12].

\section{Case Report}

An 83-year-old female presented to the emergency department with a chief complaint of abdominal pain. She reported that the pain had been persistent for approximately 2 weeks, and that it radiated to her left flank area as well as her lower back in the T12-L1 region. She described the pain as diffuse and reported a pain scale rating of 8 out of 10 . The patient reported a history of previous back surgery, anemia, and diabetes mellitus. The patient was asked if she was ever diagnosed or treated for tuberculosis (TB), and she stated that she could not remember. On physical examination, she experienced pain on light

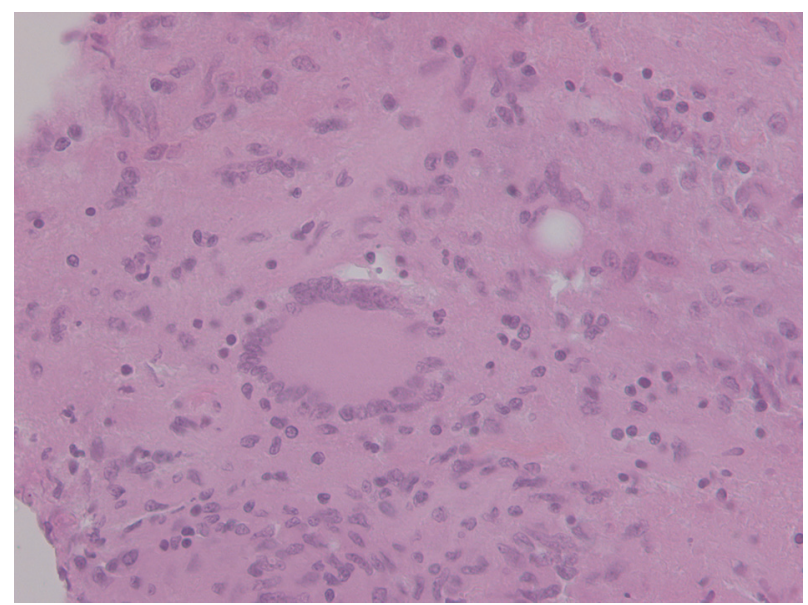

Figure 2. H\&E stain showing classic Langhans-type giant cells, epithelioid cells, and lymphocytes $(\times 40)$.

abdominal palpation, and she then refused further deep palpations of the abdomen. The patient was then admitted and a CT scan was ordered, as well as a skin TB test.

The CT imaging studies revealed two pertinent findings. The first was a large left psoas muscle complex fluid mass with calcifications measuring $7 \times 3.9 \times 2.6 \mathrm{~cm}$. The radiologic findings suggested an infectious process in which TB could not be excluded. The second major finding was severe chronic osteomyelitis/discitis involving the T11-L1 vertebral bodies. From these radiologic findings, the attending physician ordered a CT-guided biopsy of the psoas mass.

\section{Pathologic findings}

Our pathology laboratory received multiple pieces of tan-gray tissue measuring $0.8 \mathrm{~cm}$ in aggregate. H\&E-stained sections demonstrated benign striated muscle with caseating granulomatous inflammation (Fig. 1). When examined closely the histologic sections showed classic foreign body giant cells (Langhans-type) that are traditionally correlated with TB (Fig. 2). Special stains for acid fast bacilli (AFB) identified rare $M y$ cobacterial species within the granulomatous debris.

\section{Discussion}

The patient was treated medically with the accepted clinical standard protocol of isoniazid, rifampin, pyrazinamide, and ethambutol. As of October 2014, the patient was doing well and showed no signs of recurrence or complications.

TB infection involving the psoas muscle is a rare phenomenon in western industrialized countries [5]. In the past, most cases occurred in patients diagnosed with vertebral TB (Pott's disease) with subsequent spread to the psoas muscle $[5,6]$. However, TB is still widespread in developing countries and TB psoas abscesses are a frequent finding.

We report this rare case of TB presenting in the psoas muscle to make physicians and other healthcare providers aware 
of the detrimental health effects of systemic tuberculosis, and to also remind healthcare providers to be acutely aware of this rare disease when encountering patients in any healthcare setting.

\section{Financial Disclosures}

The authors have no relevant financial interest in the products or companies described in this article.

\section{References}

1. Mynter H. Acute psoitis. Buffalo Med Surg. 1881;21:202210.

2. Gruenwald I, Abrahamson J, Cohen O. Psoas abscess: case report and review of the literature. J Urol. 1992;147(6):1624-1626.

3. Ricci MA, Rose FB, Meyer KK. Pyogenic psoas abscess: worldwide variations in etiology. World J Surg. 1986;10(5):834-843.

4. Melissas J, Romanos J, de Bree E, Schoretsanitis G, Askoxylakis J, Tsiftsis DD. Primary psoas abscess. Report of three cases. Acta Chir Belg. 2002;102(2):114-117.
5. Hernandez-Ros R, Hernandez-Belmonte A. Psoas abscess: primary or secondary? Indian Pediatr. 2013;50(3):345346.

6. Mallick IH, Thoufeeq MH, Rajendran TP. Iliopsoas abscesses. Postgrad Med J. 2004;80(946):459-462.

7. Zissin R, Gayer G, Kots E, Werner M, Shapiro-Feinberg M, Hertz M. Iliopsoas abscess: a report of 24 patients diagnosed by CT. Abdom Imaging. 2001;26(5):533-539.

8. Chern $\mathrm{CH}, \mathrm{Hu} \mathrm{SC}$, Kao WF, Tsai J, Yen D, Lee CH. Psoas abscess: making an early diagnosis in the ED. Am J Emerg Med. 1997;15(1):83-88.

9. Garner JP, Meiring PD, Ravi K, Gupta R. Psoas abscess - not as rare as we think? Colorectal Dis. 2007;9(3):269274.

10. Audia S, Martha B, Grappin M, Duong M, Buisson M, Couaillier JF, Lorcerie B, et al. [Pyogenic psoas abscess: six cases and review of the literature]. Rev Med Interne. 2006;27(11):828-835.

11. van den Berge M, de Marie S, Kuipers T, Jansz AR, Bravenboer B. Psoas abscess: report of a series and review of the literature. Neth J Med. 2005;63(10):413-416.

12. Routier E, Bularca S, Sbidian E, Roujeau JC, Bagot M. [Two cases of psoas abscesses caused by group A betahaemolytic streptococcal infection with a cutaneous portal of entry]. Ann Dermatol Venereol. 2010;137(5):369-372. 